\title{
Morphologie et organisation du lexique: la catégorie nominale, comparaison des déonomastiques et des dénominaux
}

\author{
Mathilde Huguin ${ }^{1, *}$ \\ ${ }^{1}$ UMR 7118, ATILF, CNRS \& Université de Lorraine, Nancy, France
}

\begin{abstract}
Résumé. La classe nominale peut être scindée en deux : d'une part les noms communs et de l'autre les noms propres. Notre objectif est de vérifier si d'un point de vue constructionnel ces deux classes ont des comportements différents. Dans cette étude, nous comparons les Noms propres référant à des Personnalités Politiques (NPP) et les Noms communs désignant des Humains $\left(\mathrm{Nc}_{\mathrm{Hum}}\right)$. L'analyse des lexèmes construits sur $\mathrm{NPP}$ et $\mathrm{Nc}_{\mathrm{Hum}}$ nous renseigne sur les propriétés des bases. Bien que les mêmes patrons morphologiques opèrent sur les deux catégories, d'autres éléments permettent d'argumenter en faveur d'une distinction $\mathrm{Nc}_{\mathrm{Hum}} / \mathrm{NPP}$. $\mathrm{Nc}_{\mathrm{Hum}}$ et NPP possèdent des espaces thématiques au fonctionnement différent. Sémantiquement, ils ne peuvent pas non plus être caractérisés de la même manière. Si le $\mathrm{Nc}_{\text {Hum }}$ dispose d'une signification stable, le NPP possède plusieurs types de sens qui n'apparaissent qu'en contexte. Ces différences, formelles et sémantiques, confortent l'idée que la classe nominale ne peut trouver de définition unifiée. Finalement, nous mettons en évidence le fait qu'un $\mathrm{Nc}_{\mathrm{Hum}}$ n'est peut-être pas plus différent d'un NPP qu'une autre catégorie nominale (toponyme, etc.).
\end{abstract}

\begin{abstract}
Morphology and lexical organization: the nominal category, comparison of deonomastics and denominals. The nominal class can be divided in two: on the one hand the common nouns and on the other hand the proper names. Our goal is to check if from a morphological point of view these two classes have different behaviors. In this study, we compare proper names referring to political personalities (NPP) and common nouns designating humans ( $\mathrm{NcHum}$ ). The analysis of lexemes built on NPP and

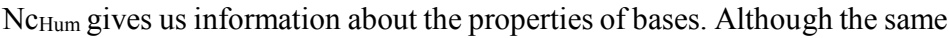
morphological patterns operate on both categories, other elements make it possible to argue in favor of a $\mathrm{Nc}_{\mathrm{Hum}} / \mathrm{NPP}$ distinction. NPP and $\mathrm{Nc}_{\mathrm{Hum}}$ have stem spaces with different functioning. Semantically, they cannot be characterized in the same way. If the $\mathrm{Nc}_{\mathrm{Hum}}$ has a stable meaning, the NPP has several types of meaning that only appear in context. These differences, formal and semantic, support the idea that the nominal class cannot find a unified definition. Finally, we highlight the fact that a $\mathrm{NcHum}_{\mathrm{c}}$ is perhaps no more different from an NPP than another nominal category (toponym, etc.).
\end{abstract}

*mathildehuguin@univ-lorraine.fr 


\section{Introduction}

Le but de cette étude est de comparer les noms propres aux noms communs. Définir ces catégories, ou trouver une limite stricte entre les deux, est compliqué. De nombreux travaux, parmi lesquels Jespersen (1924), Pulgram (1954) ou Anderson (2007) l'ont déjà souligné. Cette comparaison, déjà entamée dans la littérature (cf. §2), va être complétée par une étude morphologique. Nous nous limitons à l'étude des noms désignant des humains. Nous comparons des lexèmes construits sur: les Noms propres de Personnalités Politiques (désormais NPP) et les Noms communs désignant des Humains (désormais NcHum), respectivement illustrés par les exemples (1) et (2).

(1) MICHEL ROCARDNPr $>$ ROCARDIENAdj ${ }^{1}$

Une chose me chiffonne cependant, personne à la télé n'a soufflé un mot du passé rocardien de Manuel Valls. ${ }^{2}$

(2) BARMANNc $>$ BARMANISMENc

Et n'étant intéressé que par le «barmanisme » un BTS ne me conviendrait pas.

Notre étude se situe dans le cadre théorique de la morphologie lexématique (cf. Fradin, 2003 ; Blevins, 2006). Le lexème est une unité abstraite définie par des propriétés phonologiques, syntaxiques et sémantiques (cf. Mugdan, 2015). Une Règle de Construction de Lexème (désormais RCL) est le produit d'un ensemble de fonctions appliquées à au moins un lexème base pour élaborer un nouveau lexème. Dans notre étude, les lexèmes construits sont des déonomastiques, construits sur base NPP, et dénominaux, construits sur base $\mathrm{Nc}_{\mathrm{Hum}}$.

Dans la première partie de cet article, nous présentons les propriétés de la classe nominale telles qu'elles sont présentées dans la littérature (§2). Nous examinons ce qui permet de distinguer les noms propres des noms communs. Nous montrons pourquoi les noms propres peuvent aussi être vus comme des lexèmes. Nous caractérisons ensuite les données étudiées, dénominaux et déonomastiques, vis-à-vis du lexique (§3). Nous expliquons pourquoi et comment nous avons construit un corpus de déonomastiques. Enfin, nous comparons les déonomastiques et dénominaux d'un point de vue morphologique (§4). Nous nous attachons à décrire les RCL en jeu sur chaque type de base, les variations formelles observées et les sens des lexèmes construits.

\section{La classe nominale}

Nous débutons cette partie par l'examen des propriétés graphiques et morpho-syntaxiques généralement attribuées au nom propre en opposition au nom commun (\$2.1). La question du sens et de la référence est centrale dans l'étude qui a conduit à les distinguer (e.g. Anderson, $2007: 15)$. Les deux parties suivantes sont donc consacrées à ces propriétés $(\$ 2.2$ et §2.3). Les quelques caractéristiques constructionnelles des noms propres trouvées dans la littérature sont présentées sous $\$ 2.4$.

\subsection{Propriétés graphiques et morpho-syntaxiques : lieux communs}

Dans les grammaires d'aujourd'hui, les noms propres sont décrits de manière sporadique, à la manière d'exceptions au sein de la classe nominale (e.g. Grevisse \& Goosse, 2007). Les propriétés particulières du nom propre, quand elles ne sont pas sémantiques (cf. §2.3), sont graphiques et morpho-syntaxiques. Contrairement au nom commun, le nom propre : (a) possède une majuscule, (b) ne se met pas au pluriel, (c) n'a pas de flexion morphologique et (d) n'est ni déterminé, ni modifié. Chacune de ces affirmations est réfutable (cf. Kleiber, 1981 ; Allerton, 1987 ; Leroy, 2004 ; Vaxelaire, 2005). Nous illustrons nos propos par les exemples (3) et (4). 
(3) Cristiano Ronaldo ist zum dritten Mal Europas Fußballer des Jahres.

Cristiano Ronaldo est le footballeur européen de l'année pour la troisième fois.

(4) Marre de voir les photos retouchées et le grotesque de Brigitte Macron. Finalement, ça montre à quel point ces deux macrons sont des guignols lui avec sa com' elle avec sa vulgarite.

(a) On trouve des noms communs avec la majuscule dans d'autres langues, comme en Allemand sous (3). (b) Dans certains de leurs emplois les noms propres peuvent tout à fait être pluralisés (4) (cf. Coseriu, 1955). (c) La flexion est l'actualisation d'un lexème dans un énoncé (cf. Carstairs McCarthy, 2000 ; Stump, 2005). Le nom propre s'actualise en mots-formes en discours, il est donc sujet à la flexion. Il existe d'ailleurs des langues où les noms propres prennent les marques de cas (e.g. Grec, Russe). Le critère (c) revient sans doute à dire qu'ils ne sont pas marqués formellement lors de cette actualisation. Cette affirmation a été démentie sous (b). (d) Il existe des noms propres toujours déterminés comme Le Louvre (cf. Noailly, 1995).

Nous venons de voir que les critères graphiques et morpho-syntaxiques sont tout au plus des tendances grammaticales propres au français. Ils ne peuvent constituer une base solide de comparaison entre nom propre et nom commun.

\subsection{Propriétés référentielles}

Croft (1991), Baker (2003 : 95), Rauh (2010 : 5), parmi d'autres, soulignent le lien étroit qui unit les noms (en général) et la référence. À la suite de Lyons (1978), nous considérons que la référence est le «rapport entre une expression et ce que cette expression désigne » (op. cit. : 143). Strawson (1950) précise que la référence est effectuée par le locuteur en discours :

'Mentioning' or 'referring', is not an expression does; it is something that some one can use an expression to do. Mentioning, or referring to, something is a characteristic of a use of an expression, just as 'being about' something, and truth-or-falsity, are characteristic of a use of a sentence. (op. cit. : 326)

Le référent est « tout objet ou état de choses dans le monde extérieur qu'on peut identifier à l'aide d'un mot ou d'une expression » (Lyons, $1978: 143$ ).

Suivant ces définitions, la classe nominale peut être scindée en deux. Les noms communs désignent une classe d'individus. Les noms propres réfèrent à un individu unique. NPP comme $\mathrm{Nc}_{\text {Hum }}$ désignent des individus humains. Un NPP désigne un humain unique, identique dans tous les mondes possibles (cf. Kripke, 1980). C'est un désignateur rigide ${ }^{3}$. Un $\mathrm{Nc}_{\mathrm{Hum}}$ désigne une classe d'humains, de manière contingente. C'est un désignateur accidentel. On peut imaginer un monde où Emmanuel Macron n'est pas président mais difficilement imaginer un monde où Emmanuel Macron n'est pas Emmanuel Macron. Le président désigne actuellement Emmanuel Macron, de manière accidentelle. L'opération de référence effectuée par des individus lors d'un acte de communication est différente selon qu'il s'agit d'un NPP ou d'un $\mathrm{Nc}_{\mathrm{Hum}}$. Considérons les exemples (5) et (6).

(5) Christine Boutin s'est retirée de la politique.

(6) Le charcutier fait désormais des carottes râpées.

Pour comprendre (5), il faut avoir appris à reconnaître le référent lié au nom Christine Boutin. L'interlocuteur s'insère dans une chaine de communication où l'une des extrémités est l'acte de baptême du référent (cf. ibid.). Le nom propre nécessite une nomination préalable (cf. Kleiber, 1981). En (6), le $\mathrm{Nc}_{\mathrm{Hum}}$ fait partie d'une description définie qui peut potentiellement référer à un nombre infini de particuliers. L'interlocuteur n'est pas obligé de connaître le référent du nom commun. Une description définie peut référer à un individu inconnu, pas un nom propre. 
La première distinction entre nom propre et nom commun est de nature référentielle. Les NPP sont singularisants. Les $\mathrm{Nc}_{\mathrm{Hum}}$ désignent une classe. Le nom propre permet de différencier des individus de la même espèce. En discours, pour chacun des types de noms étudiés, l'opération mentale de référence est différente.

\subsection{Propriétés sémantiques}

Nous utiliserons sens pour parler de ce que signifie un terme en discours. La signification (ou sens lexical) est présente en langue, et résulte de l'abstraction des traits sémantiques communs aux différents sens.

\subsubsection{Traits sémantiques et classes de noms}

Contrairement aux idées reçues présentes dans les grammaires, les $\mathrm{Nc}_{\mathrm{Hum}}$ ne désignent pas nécessairement des entités concrètes, comptables, agentives et hétérogènes (cf. Schnedecker $\&$ Mihatsch, à paraître). Les $\mathrm{Nc}_{\text {Hum }}$ possèdent une dimension physique et psychologique. Cette bipartition fait qu'ils répondent négativement à des tests qui satisfont normalement les propriétés citées supra. Par exemple, les $\mathrm{Nc}_{\mathrm{Hum}}$, en tant qu'entités concrètes, ont une extension dans l'espace. Cependant, on accepte mal le syntagme ?l'extrémité d'une jeune fille contenant un nom commun locatif (cf. Flaux \& Van de Velde, $2000: 45$ ). Plusieurs traits sémantiques sont utilisés pour décrire des classes de $\mathrm{Nc}_{\mathrm{Hum}}$ (e.g. extensif/intensif ou contingent/permanent, cf. ibid.; Reboul, 1993). Ces traits caractérisent des classes telles que : noms de phase $\left(\right.$ ENFANT $_{\mathrm{Nc}}$ ), noms de spécialiste (PIANISTE $\mathrm{Nc}_{\mathrm{c}}$ ), noms de profession ( FLEURISTE $_{\mathrm{Nc}}$ ), etc. Ces classes se recouvrent et leurs frontières sont poreuses (un pianiste est un spécialiste mais aussi un professionnel cf. Lignon \& Namer, à paraître). $\mathrm{Un} \mathrm{Nc}_{\mathrm{Hum}}$ satisfait très rarement une seule classe. Il est intéressant de souligner que les NPP possèdent la même ambiguité. Un anthroponyme désigne à la fois un être physique et psychologique. Tout comme chez les $\mathrm{Nc}_{\mathrm{Hum}}$, il est difficile de trouver des traits stables qui permettraient de circonscrire la classe des anthroponymes.

\subsubsection{Définition et sens encyclopédique}

Les noms communs peuvent être définis. Cruse (1988) affirme que la signification doit contenir des informations stables relatives au mot et non déductibles de l'observation du référent. La définition sous (7), met ainsi en lien le $\mathrm{Nc}_{\mathrm{Hum}} \mathrm{MOTHER}_{\mathrm{Nc}}$ à FEMALE $\mathrm{Adj}_{\mathrm{j}}$ et PARENT $_{\mathrm{Nc}}$.

(7) Mother = 'female parent' (Cruse, $1988: 80$ )

Mère $=$ 'parent de sexe féminin'

En ce sens, la notion de signification est inopérante pour les NPP (cf. Mill, 1843). Sans information référentielle le NPP est une étiquette (PAULNPr peut être le nom d'un chat, d'un homme, d'une chaîne de boulangerie etc.). La notoriété des NPP leur confère un statut particulier en discours. Considérons l'énoncé sous (8).

(8) L'éviction de Jean-Marie Le Pen du FN est notamment due à l'action de Florian Philippot.

A priori, nous savons identifier le référent du NPP Jean Marie Le Pen. Nous disposons de connaissances, variables selon les individus, vis-à-vis de ce référent. Ces deux mécanismes, d'ordre pragmatique plus que sémantique, sont schématisés par la Figure 1. 


\section{Jean-Marie Le Pen}

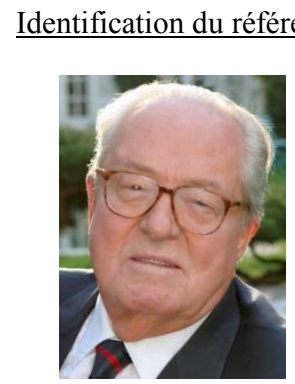

$$
\begin{gathered}
\frac{\text { Convocation de connaissances vis-à-vis du }}{\text { référent }} \\
\text { Condamné pour racisme, incitation à la haine, } \\
\text { antisémitisme, négationnisme... } \\
\text { Fondateur du Front National } \\
\text { Père de Marine Le Pen } \\
\text { Nationaliste } \\
\text { Député européen } \\
\text { Engagé pendant la guerre d'Algérie } \\
\text { Président d'honneur du Front National... }
\end{gathered}
$$

Fig 1. Mécanismes pragmatiques à l'œuvre lors de l'apparition d'un NPP en contexte : l'exemple de Jean-Marie Le Pen.

Ces mécanismes peuvent échouer si le lecteur ne s'inscrit pas dans la communauté linguistique concernée. Gary-Prieur $(1994,2016)$ théorise cette bipartition à l'intérieur du sens de l'anthroponyme. D'une part l'anthroponyme dispose de ce que Kleiber (1999) nomme un sens dénominatif instructionnel. À la manière d'un déictique comme je ou ici, il faut identifier le référent correspondant à l'étiquette NPP. D'autre part, l'anthroponyme dispose d'un contenu. Il s'agit d'informations encyclopédiques relatives au référent (on retrouve cette idée chez de nombreux auteurs e.g. Allerton, 1987 ; Langendonck, 2007 ; Anderson, 2007 ; McKinsey, 2010). Le Tableau 1, ci-dessous, schématise notre propos.

Tableau 1. La bipartition sémantique de l'anthroponyme : l'exemple de CHRISTINE BOUTINNPr.

\begin{tabular}{|l|l|}
\hline $\begin{array}{l}\text { Sens dénominatif } \\
\text { instructionnel }\end{array}$ & Individu porteur du nom propre Christine Boutin \\
\hline $\begin{array}{l}\text { Connaissances } \\
\text { encyclopédiques }\end{array}$ & $\begin{array}{l}\text { Femme politique française de droite, prônant des valeurs catholiques, } \\
\text { mariée à son cousin germain... }\end{array}$ \\
\hline
\end{tabular}

Les NPP et $\mathrm{Nc}_{\mathrm{Hum}}$ sont donc différents d'un point de vue sémantique. Les $\mathrm{Nc}_{\mathrm{Hum}}$ possèdent une signification stable. Les NPP n'ont pas de signification. En discours, ils déclenchent deux types de mécanismes pragmatiques qui donnent accès à deux types de sens. Le premier mécanisme est instructionnel, nous devons identifier le référent. Le second est encyclopédique, l'identification du référent convoque des connaissances liées à la personnalité politique.

\subsection{Propriétés constructionnelles}

\subsubsection{Nchum et NPP sont-ils morphologiquement construits ?}

Certains $\mathrm{Nc}_{\mathrm{Hum}}$ sont morphologiquement construits (9) mais pas tous (10) (cf. Marin \& Villoing, 2012 ; Tribout \& al., 2014).

(9) VIOLONNc $>$ VIOLONISTENc

(10) FEMMENc

Parfois les $\mathrm{Nc}_{\mathrm{Hum}} \mathrm{s}$ 'insèrent dans une famille dérivationnelle où la base n'est plus identifiable. La base peut être, comme sous (11), démotivée en synchronie.

(11) CHARCUTIERNc $_{\mathrm{Nc}}$, CHARCUTIÈRE $\mathrm{Nc}$, CHARCUTERIE ${ }_{\mathrm{Nc}}$

Il est toujours possible de créer des $\mathrm{Nc}_{\mathrm{Hum}}$ (e.g. nouveaux métiers : DRONISTENc, 'pilote de drone professionnel'). Les $\mathrm{Nc}_{\mathrm{Hum}}$ sont donc une classe ouverte. 
Les anthroponymes français sont composés de deux parties, le prénom et le patronyme. Par la suite, nous utiliserons anthroponyme pour parler de l'ensemble prénom et patronyme. Tribout \& al. (2014) propose qu'un mot construit : (i) soit interprétable à partir d'un autre lexème de la langue en synchronie ; (ii) corresponde au résultat d'une RCL opérant en français. Observons les exemples sous (12) en parallèle de la définition de mot construit.

(12) STRAUSS-KAHNNPr ; JEAN-PIERRENPr; GEORGETTENPr ; NICOLAS SARKOZYNPr

Ces exemples soulèvent des problèmes peu abordés en morphologie synchronique ( $v s$ onomastique e.g. Hough \& Izdebska, 2016). Les lexèmes sous (12) sont-ils le résultat de constructions morphologiques ? Dans Lipka (2000), les anthroponymes sont analysés comme des cas de composition. L'auteur analyse également les séries de patronymes sous (13) comme des compositions où les éléments $O$ '- et $M c$ - apportent le sens de 'fils de'.

(13) O'Hara, McArthur, McDonald...

Ces données poussent l'auteur à affirmer à propos des noms propres :

Often they are clearly morphologically or semantically motived, a fact that is not recognised in familiar, habitualised, famous names, e.g., Richard Wargner. They combine various productive word-formation processes, such as compounding, derivation, clipping, blending and acronyming. (op. cit. : 200)

Néanmoins, le NPP étant sémantiquement vide hors contexte on ne peut pas interpréter JEAN-PIERRENPr à l'aide des lexèmes JEAN NPr $_{\text {r }}$ et PIERRENPr. Dans plusieurs ethnies africaines et en Langue des Signes Française, l'anthroponyme est sémantiquement motivé par une caractéristique du référent (cf. Journet-Diallo, 2001). Ce sont des aptonymes. Si c'était également le cas en France il y a plusieurs centaines d'années, les anthroponymes semblent aujourd'hui majoritairement démotivés (e.g. LEFEBVRE $\mathrm{NPr}_{\mathrm{r}}$ vient de FORGERON $\mathrm{N}_{\mathrm{Nc}}$ ). Le premier critère de Tribout \& al. (2014) est inopérant. Il faut donc se tourner vers le deuxième critère. On peut analyser formellement JEAN-PIERRE ${ }_{\mathrm{NPr}}$ comme un cas de composition de deux noms. Ce procédé existe également chez les $\mathrm{Nc}_{\mathrm{Hum}}$ (e.g. HOMME-GRENOUILLE $\mathrm{Nc}_{\mathrm{Nc}}$ ). Ce procédé est disponible puisque l'on rencontre de nouveaux prénoms composés tous les ans (e.g LILY-ROSENPr). La classe des noms propres, comme celle des noms communs, est donc ouverte. Ces éléments nous poussent à analyser certains NPP comme construits.

Les $\mathrm{Nc}_{\mathrm{Hum}}$ peuvent être construits ou non. On vient de voir que pour les NPP, la situation est moins tranchée. Formellement, plusieurs cas laissent à penser qu'ils sont construits et/ou motivés. La question de catégoriser l'anthroponyme dans sa globalité comme construit ou non demanderait à être étudiée en détail.

\subsubsection{Les déonomastiques et dénominaux}

En français, il existe peu d'études consacrées à l'analyse morphologique des déonomastiques (on peut citer la monographie conséquente de Schweickard, 1992 et les travaux de Leroy, 2005, 2008). Ils apparaissent pourtant dans de nombreuses études (cf. (14) à (16)), souvent à titre d'exemple.

(14) HONORÉ DE BALZACNPr > BALZACIENAdj (Lignon, $2002: 2$ )

(15) AMÉLIE POULAINNPr > AMÉLIEPOULINISERv (Namer, 2013)

(16) NICOLAS SARKOZYNPr > SARKOZYCRATIENc (Lasserre, $2016: 172$ )

Il semblerait que les noms propres et déonomastiques disposent de caractéristiques constructionnelles spécifiques. Anderson (2007), par exemple, affirme que les noms propres possèdent des marqueurs propres, i.e. certains affixes leurs sont réservés (e.g. suffixation en -ský en polonais). Et sémantiquement, les déonomastiques convoquent en construction un savoir encyclopédique sur le référent (e.g. sadisme, op. cit. : 328). Le travail engagé dans 
Leroy (2008) révèle que certains affixes français sont réservés aux toponymes pour construire des gentilés (e.g. -at: AUVERGNAT $\mathrm{N}_{\mathrm{Nc}}$ ). L'auteur ne trouve pas d'affixes spécifiques aux anthroponymes.

À l'inverse les travaux sur le nom commun et les dénominaux sont très nombreux. Nous utiliserons les résultats existants pour établir une comparaison entre déonomastiques et dénominaux.

\subsection{Bilan}

Nous avons vu qu'il existe des différences entre NPP et $\mathrm{Nc}_{\mathrm{Hum}}$ d'un point de vue référentiel et sémantique. Les NPP réfèrent à un individu unique, les $\mathrm{Nc}_{\mathrm{Hum}}$ désignent une classe. Les $\mathrm{Nc}_{\mathrm{Hum}}$ disposent d'une signification. En contexte, les NPP font appel à des mécanismes d'ordre pragmatique. Ils disposent alors d'un sens dénominatif instructionnel et d'un contenu. Les critères constructionnels ne sont pas utilisés pour appuyer la délimitation d'une catégorie. La littérature ne fait pas état de différence significative au niveau morphologique. Alors que les $\mathrm{Nc}_{\mathrm{Hum}}$ peuvent être construits ou non, la réponse est plus difficile à formuler pour les NPP.

Dans la suite, nous considérerons les noms propres comme des lexèmes à part entière. Effectivement, nous avons précisé qu'ils constituaient une liste ouverte (cf. \$2.4.1) et nous ajoutons qu'ils peuvent être la tête d'un syntagme de même catégorie (cf. Allerton, 1987 ; Matushansky, 2008). La catégorie nom propre répond donc positivement aux caractéristiques des catégories lexicales proposées dans Fradin (2003: 89).

Dans notre étude, nous comparons des dénominaux et des déonomastiques. Nous cherchons à savoir si la morphologie peut apporter des éléments pour mieux définir les noms communs et les noms propres. Peut-on trouver des différences suffisantes pour identifier deux catégories partitionnant clairement la classe nominale ? La section suivante présente les données recueillies.

\section{Présentation des données}

Dans cette section nous expliquons pourquoi nous avons constitué notre propre corpus de déonomastiques (\$3.1) et présentons la méthodologie adoptée (§3.2).

\subsection{Statut des déonomastiques dans le lexique}

Si l'accès aux dénominaux est aisé, à partir des dictionnaires (e.g. TLF) ou des corpus existants (e.g. Lexique 3), il n'en n'est pas de même pour les déonomastiques. Nous avons choisi de travailler uniquement sur les construits prenant pour base des NPP contemporains (e.g. EMMANUEL MACRON $\mathrm{N}_{\mathrm{NP}}$ ). La nature contemporaine de ces bases fait que les mots qui en dérivent sont souvent absents des dictionnaires. Ces déonomastiques ne sont pas lexicalisés et n'ont pas nécessairement vocation à le devenir (cf. Hohenhaus, 2005). On peut les qualifier, à la suite de Dal \& Namer (2016), d'occasionnalismes. Ils servent à « satisfaire un besoin immédiat dans une situation communicationnelle donnée » (op. cit. : 2). Ces construits résultent par exemple d'une " pulsion ludique » (cf. Lipka, 2000) et s'insèrent dans des jeux de mots et figures de style. Dans l'exemple (17), les jeux à partir des syntagmes « école buissonnière » et « écriture italique » sont basés sur une ressemblance phonologique.

(17) ERIC BESSONNPr $>$ BESSONIĖREAdj JACQUES ATTALINPr $>$ ATTALIQUEAdj

De maroquins en commissions, l'école bessonière connut alors son heure de gloire au point que les rapports furent écrits en attalique, cette écriture qui penche singulièrement à droite.

Les caractéristiques que nous venons de décrire rendent difficile l'accès aux construits sur NPP. Ceci nous a poussée à créer notre corpus de déonomastiques. La prochaine section explique le protocole méthodologique mis en place pour construire ce corpus. 


\subsection{Création de la base de données lexicale}

Nous avons sélectionné 90 NPP dont les référents ont exercé une fonction politique importante depuis $1981^{4}$. Nous avons ensuite généré des formes candidates à partir de ces NPP et de séquences identifiées comme apparaissant à droite d'un NPP. Ce sont majoritairement des suffixes et des exposants de composition néoclassique, mais pas seulement, puisque les désinences de l'infinitif(e.g. -er) sont représentées. La démarche pour fabriquer ces candidats est la suivante : nous trions les déonomastiques extraits du $\mathrm{frWaC}$ (l'exemple (18) illustre ce que l'on obtient à partir de Sarkozy, sous la forme sarko*5), nous isolons ensuite les séquences finales, comme présenté sous (19).

(18) sarkoziste, sarkosisme, sarkozienne, sarkozien, sarkophage, sarkozique, sarkozie, sarkosphère, sarkolâtrie, sarkozerie, sarkophobie, sarkomania, sarkothon, sarkozix

(19) -iste, -isme, -ienne, -ien, -phage, -ique, -ie, -sphère, -lâtrie, -erie, -phobie, -mania, -thon, -ix

En réitérant cette démarche à partir de 15 NPP nous avons collecté 103 séquences finales.

Dans l'élaboration de l'algorithme de génération automatique des candidats, nous avons pris en compte la pression des contraintes morpho-phonologiques et graphiques existantes en français (cf. Lignon \& Roché, 2011; Villoing, 2012 ; Plénat \& Roché, 2014). Ces contraintes, souvent antagonistes, entraînent des variations sur la forme de sortie possible, i.e. la forme candidate. En (20) b) et c), on envisage une haplologie pour éviter la succession des phonèmes similaires /i/ présents dans la base et l'affixe (cf. Plénat, 2011).
(20) MARTINE AUBRYNPr > a) aubryiste
b) aubriste
c) aubryste

(21) est un échantillon de formes candidates générées à partir de CHRISTIANE TAUBIRANPr.

(21) taubiralisme, taubiresque, taubirologue, taubiracratie, christianien, christianetaubirique

Ces formes sont utilisées comme requêtes par une application qui appelle le moteur de recherche Bing (Bing Search API). La collecte est effectuée en partenariat avec l'entreprise Data-Observer ${ }^{6}$. Tous les contextes des formes candidates sont relevés.

\subsection{Bilan}

Les déonomastiques issus de NPP ont un caractère volatile et sont absents des dictionnaires. Leur création résulte rarement d'un besoin dénominatif. Ces propriétés nous ont poussée à créer notre propre corpus de déonomastiques. Les formes collectées sont organisées sous la forme d'une base de données où chaque entrée décrit la relation entre une base NPP et un lexème construit. Notre base de données contient 5550 lexèmes et 55262 occurrences.

Notre objectif est de comparer les propriétés morphologiques des NPP et $\mathrm{Nc}_{\mathrm{Hum}}$. La section suivante résulte de l'analyse des construits sur chaque type de base et donne les premiers résultats de cette comparaison.

\section{Comparaison des déonomastiques et des dénominaux}

La première partie de cette section propose un bilan général des RCL opérant sur NPP et $\mathrm{Nc}_{\text {Hum }}(\S 4.1)$. La seconde décrit les conditions formelles de construction des dénominaux et déonomastiques (\$4.2). Enfin, la troisième partie compare les mécanismes en jeu lors de la sélection sémantique du NPP et du $\mathrm{Nc}_{\mathrm{Hum}}$ par la RCL (§4.3). 


\subsection{Remarques générales : à propos des RCL en jeu}

Nous montrons dans cette première partie que $\mathrm{Nc}_{\mathrm{Hum}}$ et NPP sont sélectionnés comme base par les mêmes RCL. Déonomastiques et dénominaux diffèrent cependant sur plusieurs points, notamment en termes de connotation (au sens de Gary-Prieur, 1971).

\subsubsection{Points communs}

$\mathrm{NPP}$ et $\mathrm{Nc}_{\mathrm{Hum}}$ construisent des lexèmes appartenant aux trois classes sémantiques majeures : actions (22), objets (23), et propriétés (24) (cf. Croft, 1991 ; Flaux \& Van de Velde, 2000) et aux trois classes lexicales : verbes, noms et adjectifs.

(22) a. Plus qu'un bazooka, interpelle nos élus, pourquoi ils ont laissé une jeunesse se crapuliser. b. François Fillon doit élargir sa base électorale sans renier ce qu'il est, sans se juppéiser.

(23) a. Vu sur eBay, le Gamerator a pour particularité d'être une borne d'arcade associée à un bar.

b. Cent jours de présidence : avec notre hollandoscope, vérifiez si Hollande tient ces promesses.

(24) a. Jeste ar Manan, c'était le plus crapuleux, mis à la porte de la marine, de la coloniale, des Bat d'Af, il finit à la légion étrangère.

b. Avec un nom pareil, il est taubirable, donc libérable aussitôt.

Dans notre corpus, toutes les RCL opérant sur NPP semblent opérer sur $\mathrm{Nc}_{\mathrm{Hum}}$. Nous affirmons qu'il s'agit des mêmes RCL car les niveaux formels, catégoriels et sémantiques du lexème base subissent la même modification. Citons par exemple la construction d'adjectifs relationnels en -ien (25) (déjà étudiés dans Lignon, 2000), ou la suffixation en -ite créant des noms de maladie (26).

(25) a. [...] ses crises d'angoisse, et son côté torturé qui, selon moi, va au-delà du simple malaise adolescentien [...].

b. Les syndicats étant passés successivement de la défense des travailleurs opprimés au sens arlettien du terme, à la défense des salariés du secteur secondaire [...].

(26) a. La vasectomie en effet c'est pratique comme solution, mais radical ! La bébéite aiguë je me demande si on ne l'a pas toutes.

b. Vous ne seriez pas en train de nous couver une petite bayroutite aiguë, par hasard ?

Des RCL s'appliquant sur d'autres catégories lexicales opèrent sur NPP. Ce point commun fournit un argument supplémentaire pour analyser le nom propre comme lexème (cf. §2.5).

\subsubsection{Différences}

Nous avons vu que les déonomastiques sont rarement lexicalisés (cf. §3.1). Cette instabilité conduit les locuteurs à créer une pluralité de déonomastiques pour désigner la même chose, en utilisant des suffixes concurrents (cf. Lignon, 2002 sur la concurrence affixale). C'est la première différence entre $\mathrm{Nc}_{\mathrm{Hum}}$ et $\mathrm{NPP}$. Ce phénomène existe sur $\mathrm{Nc}_{\mathrm{Hum}}$ mais il est plus rare car les dérivés sont souvent déjà lexicalisés. Pas moins de 17 lexèmes formellement différents, construits sur FRANÇOIS BAYROU $\mathrm{NPr}_{\mathrm{N}}$, désignent des partisans :

(27) BAYROLISTENc, BAYROUDEUSENc, BAYROUIENNENc, BAYROUISTENc, BAYROULÂTRENc, BAYROULETTENc, BAYROULIENNc, BAYROUMANIAQUENc, BAYROUNISTENc, BAYROUPHILENc, BAUROURISTENc, BAYROUSIEN $\mathrm{Nc}_{\mathrm{N}}$, BAYROUSISTENc, BAYROUTEUX $\mathrm{Nc}_{\mathrm{N}}$, BAYROUZIEN $\mathrm{N}_{\mathrm{N}}$, FRANÇOISBAYROUTISTENc, BAYROUIENNc

(28) Tes trépidations de jeune bayroliste indigné par « l'immoralité » du monde n'y changeront rien. 
La seconde différence observée est d'ordre morpho-pragmatique. Elle concerne la connotation des lexèmes construits. Les référents de NPP sont plus exposés aux jugements que les référents de $\mathrm{Nc}$, désignant des classes et non des individus. Cette caractéristique pousse les locuteurs à créer des lexèmes porteurs d'une valeur axiologique, souvent péjorative, comme sous (29). En comparaison, l'exemple sous (30), construit avec la même RCL sur base $\mathrm{Nc}_{\mathrm{Hum}}$ a une connotation neutre : 'faire l'huissier'.

(29) Mais va te faire taubiraniser putain !

(30) Oh mon dieu, et moi qui part de chez vous le 14 août au soir je ne pourrai pas huissiériser la fin du concourt.

En prenant en compte les néologismes à base $\mathrm{Nc}_{\mathrm{Hum}} \mathrm{du}$ fr $\mathrm{WaC}$, nous n'avons pas trouvé de RCL s'appliquant uniquement aux NPP. Des travaux comme Schulcket \& Ackermann (2017) ont montré que, dans d'autres langues, il existait des affixes réservés aux noms propres. Il serait intéressant de savoir si des données non lexicalisées, comme des occasionnalismes, ont été prises en compte. Finalement, nous avons dégagé deux différences entre $\mathrm{Nc}_{\mathrm{Hum}}$ et NPP. Il existe une multitude de déonomastiques concurrents dans les familles de NPP et, les RCL quand elles opèrent sur NPP, sont souvent porteuses d'une valeur axiologique.

\subsection{Au moins deux organisations thématiques nominales}

Nous considérons que les NPP sont des lexèmes (cf. §2.5). À la suite de Bonami \& Boyé (2003), Bonami, Boyé \& Kerleroux (2009) nous appelons espace thématique la composante formelle des lexèmes. Un espace thématique est un ensemble de plusieurs thèmes qui entretiennent des liens de dépendance réguliers. Alors que pour les verbes et pour les adjectifs cet ensemble est relativement bien défini et délimité, la notion est à l'étude pour les noms communs (cf. Plénat, 2008 ; Boyé \& Plénat, 2014).

Plénat \& Roché (2014) propose que l'espace thématique nominal soit divisé comme suit : (i) le thème A ou 1 correspond à la forme libre, il peut, très rarement, $\mathrm{y}$ avoir un thème pluriel (normalement réservé à la flexion, grisé dans le tableau); (ii) le thème $\mathrm{B}$ ou 2 est celui réservé aux suffixations populaires et aux conversions ; (iii) le thème $\mathrm{S}$ est le thème savant, issu du latin ou du grec, il est normalement sélectionné pour les formations savantes. Le Tableau 2 illustre les propositions de Plénat \& Roché (2014).

Tableau 2. Espace thématique des noms communs? ${ }^{7}$.

\begin{tabular}{|c|c|c|c|c|c|c|}
\hline & \multicolumn{2}{|c|}{ Thème 1} & Thème 2 & \multicolumn{3}{|c|}{ Thème savant } \\
\hline CHEVAL & $\begin{array}{l}\text { / Jəval/ } \\
\text { CHEVAL }\end{array}$ & 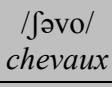 & $\begin{array}{c}\text { / Jəval/ } \\
\text { CHEVALIER }\end{array}$ & $\begin{array}{c}/ \mathrm{kaval} / \\
\text { CAVALIER }\end{array}$ & $\begin{array}{c}\text { lip/ } \\
\text { HIPPIQUE }\end{array}$ & $\begin{array}{c}/ \varepsilon \mathrm{k} / \\
\text { EQUESTRE }\end{array}$ \\
\hline EAU & $\begin{array}{l}10 / \\
\text { EAU }\end{array}$ & & $\begin{array}{c}/ \mathrm{o} / \\
\text { CHAUFFE-EAU }\end{array}$ & $\begin{array}{c}\text { /ak/ } \\
\text { AQUEDUC }\end{array}$ & $\begin{array}{c}\text { /idR/ } \\
\text { HYDRIQUE }\end{array}$ & \\
\hline BRIQUE & $\begin{array}{l}\text { /brik/ } \\
\text { BRIQUE }\end{array}$ & & $\begin{array}{l}\text { /brikət/ } \\
\text { BRIQUETER }\end{array}$ & & & \\
\hline ENFANT & $\begin{array}{c}\text { /ãfã// } \\
\text { ENFANT }\end{array}$ & & $\begin{array}{c}\text { /ãfãt/ } \\
\text { ENFANTIN }\end{array}$ & 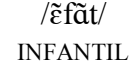 & $\begin{array}{l}\text { /pyer/ } \\
\text { PUÉRIL }\end{array}$ & $\begin{array}{c}/ \mathrm{p} \varepsilon \mathrm{d} / \\
\text { PÉDIATRE }\end{array}$ \\
\hline
\end{tabular}

Ce tableau illustre les problèmes liés à l'espace thématique des noms communs. Les thèmes des noms communs : (i) ne sont pas prévisibles, tant pour ce qui est de leur nombre que de leur forme, (ii) n'entretiennent pas de relation de dépendance, (iii) ne sont pas nécessairement sélectionnés par une règle flexionnelle ou dérivationnelle en particulier. Autrement dit, il semble difficile de dresser une organisation thématique systématique pour les $\mathrm{Nc}_{\text {Hum. }}$. 
À notre connaissance, il n'existe aucun travail sur la structure formelle des NPP. Considérons (31) à (33), contenant des lexèmes construits sur MARTINE AUBRY $\mathrm{NPr}_{\mathrm{N}}$.

(31) La « martinomania » n'est pas encore un capital à rente garantie.

(32) L’aubrisme ça nous a enfumés trop longtemps !

(33) J'ai regardé à la télévision hier après-midi Martine Aubry qui présentait ses vœux à la presse et j'ai chopé du coup peu après la fin du discours une martinaubrite.

Toutes les parties de l'anthroponyme peuvent être utilisées comme radical : prénom (31), patronyme (32) et anthroponyme (33). Les NPP disposent d'un espace formel contenant plusieurs cases et chacune correspond à une dénomination du référent. Notre proposition est illustrée dans le Tableau 3. Si l'anthroponyme est composé, comme sous (b), trois cases supplémentaires sont ajoutées : les deux parties de la composition et un sigle ${ }^{8}$.

Tableau 3. Espace thématique des anthroponymes.

\begin{tabular}{l|l|l|l|l|l|l|l}
\multicolumn{2}{l|}{} & Prénom & Patronyme & PrénomPatronyme & Comp1 & Comp2 & Sigle \\
\hline (a) & $\begin{array}{l}\text { MARTINE } \\
\text { AUBRY }\end{array}$ & /maRtin/ & /obri/ & /maRtinobri/ & & & \\
\hline (b) & $\begin{array}{l}\text { DOMINIQUE } \\
\text { STRAUSS-KAHN }\end{array}$ & $/$ dominik/ & /stroskan/ & /dominikstroskan/ & $/$ stros/ & $/$ kan/ & $/$ decska/
\end{tabular}

Contrairement aux $\mathrm{Nc}_{\mathrm{Hum}}$, tous ces thèmes sont déductibles de la forme de base du NPP. Les thèmes entretiennent un lien de dépendance et tous peuvent être utilisés en flexion. Ils sont syntaxiquement autonomes.

On peut se demander si cet espace est généralisable à tous les noms propres. Ce n'est pas le cas puisque, par exemple, le comportement des toponymes est plus proche de celui des noms communs. Eux-aussi disposent, éventuellement, d'un radical savant (34). Cette caractéristique est liée au caractère presque compositionnel de certains toponymes. NEUVE-MAISON $_{N P r}$ pourrait s'interpréter formellement comme une composition de $\mathrm{NEUF}_{\text {Adj }}$ et MAISON $_{\mathrm{Nc}}$, tous deux disposant de thèmes savants en français. Aucune règle ne sélectionne le premier ou second composant du toponyme comme c'est le cas chez les NPP (e.g. néodomien vs ${ }^{\circ}$ neuvien).

(34) NEUVE-MAISONNPr > NÉODOMIENNc Radical : /neodom/

Les NPP sont donc différents des $\mathrm{Nc}_{\text {Hum }}$ au niveau formel. Définis comme lexèmes, le fonctionnement de leurs espaces thématiques est différent.

\subsection{Sélection sémantique et polysémie}

Nous avons vu que NPP et $\mathrm{Nc}_{\mathrm{Hum}}$ diffèrent sur le plan sémantique (cf. §2.3). A l'inverse des $\mathrm{Nc}_{\text {Hum, }}$ les NPP n'ont pas de signification. Ils enclenchent des mécanismes pragmatiques lors de leur actualisation. Nous cherchons à savoir quelles sont les propriétés sémantiques de la base que l'on retrouve dans les construits. Nous appuierons notre propos sur l'analyse des exemples (35) à (39). Nous proposons une glose de chaque dérivé sous la forme = 'glose'.

La majorité des dénominaux s'interprète grâce à la signification du $\mathrm{Nc}_{\mathrm{Hum}}$ base, sans informations référentielles, comme sous (35) et (36).

(35) ADOLESCENTNc $>$ ADOLESCENTISTEAdj

$=$ 'd'adolescent'

Tandis que la droite radicale a une vision adolescentiste de la politique [...].

(36) CHAUFFEUR $\mathrm{Nc}_{\mathrm{c}}>$ CHAUFFEURISERV

= 'faire le chauffeur (pour X)'

Je suis amené à « chauffeuriser » des personnalités dans de luxueuses et puissantes voitures. 
Pour interpréter les déonomastiques, le sens de la base NPP intervenant dans le construit peut être dénominatif, comme en (37).

(37) CHRISTINE BOUTINNPr $>$ BOUTINIENAdj = 'de /Christine Boutin/'

J'ai entendu cette femme sur France-info : je ne pense pas qu'elle réclame l'euthanasie de gaité de cœur, et quand elle dit que la souffrance est intolérable et que plus rien ne la soulage, j'ai plus tendance à la croire qu'à acquiescer à la rhétorique boutinienne.

Pour interpréter pleinement le sens de certains énoncés contenant des déonomastiques, il est nécessaire de convoquer des connaissances encyclopédiques et stéréotypiques liées au référent. En (38), le construit peut s'interpréter comme 'homophobe' ou 'transphobe'. Effectivement, Conchita Wurst est une drag Queen et Christine Boutin est une femme de droite, notamment condamnée pour homophobie. A ces connaissances sur le référent s'ajoute une dimension axiologique péjorative. Le locuteur semble juger les idées de Christine Boutin détestables (cf. Roché, 2011 : 72).

(38) CHRISTINE BOUTINNPr > CHRISTINEBOUTINISTEAdj = 'homophobe, transphobe'

Si je ne trouve pas que \#ConchitaWurst a fait la meilleure prestation de l'Eurovision, ça fait de moi une fasciste christineboutiniste ?

En (39), en plus de convoquer des connaissances sur Ségolène Royal, le sens du construit sélectionne un ensemble de propriétés prototypiques du référent relativement à des évènements précis.

(39) SÉGOLÈNE ROYALNPr > SÉGOLÈNISERV $=$ 's'excuser sans raison',

Sans vouloir « ségolèniser », je présente mes excuses à mon lectorat très nombreux en cette période pré-électorale...

Notons que BALKANISATION $\mathrm{Nc}_{\mathrm{Nc}}$, construit sur un toponyme, s'interprète également grâce à des connaissances extra-linguistiques comme 'un processus similaire aux événements géopolitiques advenus dans les BALKANSNPr'.

La pragmatique joue donc un rôle important lors de la construction de déonomastiques. Cette analyse sémantique confirme la bipartition du sens des NPP proposée sous §2.3.2. La Figure 2 illustre notre propos. Une RCL opérant sur NPP peut sélectionner un sens dénominatif ou une/des informations(s), objectives ou subjectives, à propos du référent.

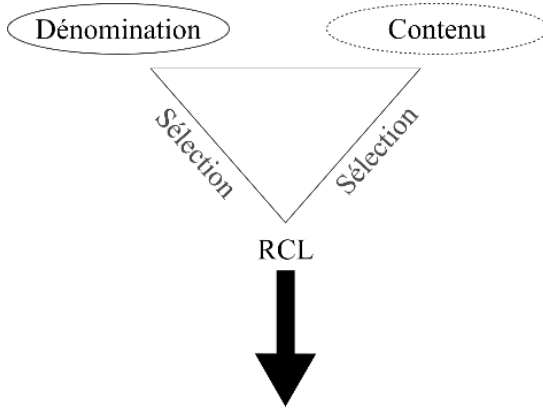

Déonomastique

Fig. 2. Sélection sémantique d'une RCL lors de la construction d'un déonomastique.

Il est difficile de qualifier le lien entre dénomination et contenu. Ce lien ne relève pas d'un mécanisme sémantique mais plutôt d'une expérience extra-linguistique. Le locuteur peut apprendre des informations sur un NPP (contenu) qu'il sait déjà identifier (dénomination) ou, connaître un référent sans pour autant le nommer. Il n'y a pas de dérivation d'un sens vers l'autre contrairement aux $\mathrm{Nc}_{\mathrm{Hum}}$ polysémiques. Les $\mathrm{Nc}_{\mathrm{Hum}}$ polysémiques disposent de deux significations. CONCIERGE ${ }_{\mathrm{Nc}}$ possède deux acceptions a) et b) proposées sous (40). 
(40) a) Personne qui a la garde d'un immeuble.

b) Personne qui aime les potins, les commérages.

Il s'agit de polysémie régulière (cf. Apresjan, 1974; Barque, 2008: 79). Les deux significations sont dérivables par des mécanismes sémantiques reproduits par ailleurs dans le lexique. Il existe effectivement d'autres noms qui établissent un lien entre un métier et l'attitude prototypique de la personne qui exerce ce métier (e.g. BOUCHER ${ }_{\mathrm{Nc}}$, ORFÈVRE $\mathrm{Nc}_{\mathrm{Nc}}$ ). La RCL pourra donc opérer sur l'une ou l'autre de ces significations. (41) est construit sur la signification a) et (42) construit sur b).

(41) CONCIERGENc $>$ CONCIERGERISMENc $=$ 'attitude de raconter des potins'

Je n'ai pas besoin d'aller récolter des infos sur le web vous concernant... Car vous étalez tout ici de vous-même par secretstorytellisme sans doute... Et ce serait le comble du comble de me le reprocher, car c'est votre passe-temps favoris le conciergerisme...

(42) CONCIERGENc $>$ CONCIERGERIENc = 'lieu où travaille le concierge'

Les derniers reflets des pelouses, une corbeille de fleurs, des arbustes taillés précédaient une conciergerie. (TLFi, MALĖGUE, Augustin, t. 1, 1933, p. 146.)

(41) et (42) sont construits sur deux bases différentes CONCIERGE $_{\mathrm{Nc} 1}$ et CONCIERGE Nc2. $_{\text {. }}$ BOUTINIEN $_{\text {Adj }}$ (37) et CHRISTINEBOUTINISTEAdj (38) sont construits sur la même base CHRISTINE BOUTIN $_{\mathrm{NPr}}$. Finalement, la sélection sémantique de la RCL n'opère pas au même niveau chez $\mathrm{Nc}_{\mathrm{Hum}}$ et NPP. La RCL sélectionne une signification stable sur $\mathrm{Nc}_{\mathrm{Hum}}$. Elle sélectionne un sens, variable en fonction du contexte d'énonciation, dans le cas des NPP. La Figure 3 schématise la sélection sémantique d'une RCL. Nous y comparons les mécanismes en jeu sur $\mathrm{Nc}_{\text {Hum }}$ polysémique et NPP.

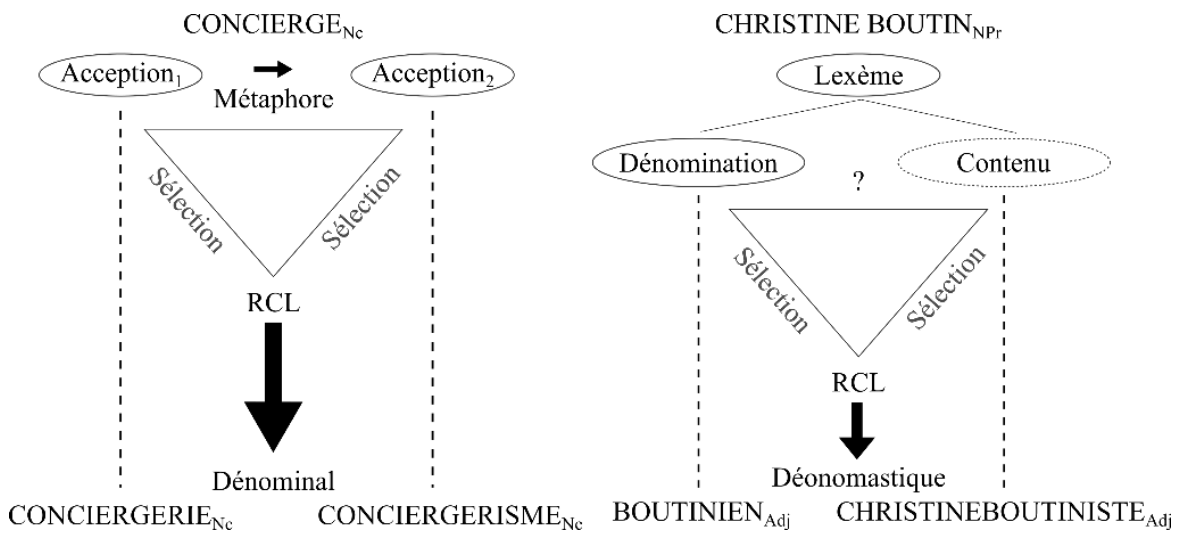

Fig. 3. La sélection sémantique lors de l'application d'une RCL : comparaison $\mathrm{Nc}_{\mathrm{Hum}}$ polysémique/NPP.

\section{Conclusion}

Notre but était de montrer que l'analyse morphologique des déonomastiques et dénominaux apporterait des arguments concernant la distinction entre nom propre et nom commun. Dans l'état de l'art, nous avons vu que seuls des critères sémantiques et référentiels distinguent $\mathrm{Nc}_{\mathrm{Hum}}$ et NPP. Nous avons affirmé que les noms propres sont une catégorie lexicale et donc des lexèmes en tant que tels.

Lors de notre analyse, nous avons comparé les RCL en jeu sur NPP et $\mathrm{Nc}_{\mathrm{Hum}}$. Toutes les $\mathrm{RCL}$ opérant sur NPP dans notre corpus opèrent sur $\mathrm{Nc}_{\mathrm{Hum}}$. Appliquées aux NPP, les RCL sont plus souvent porteuses d'une valeur axiologique péjorative. Finalement, on peut se 
demander si trouver des RCL différentes sur NPP ou $\mathrm{Nc}_{\mathrm{Hum}}$ fournirait un argument pour distinguer les noms propres des noms communs.

La comparaison formelle des dénominaux et déonomastiques nous a permis de définir deux organisations thématiques différentes pour $\mathrm{Nc}_{\mathrm{Hum}}$ et NPP. Le fonctionnement de l'espace thématique des NPP n'est pas généralisable à l'ensemble des noms propres mais il l'est pour l'ensemble des anthroponymes.

Les différences sémantiques des $\mathrm{Nc}_{\mathrm{Hum}}$ et NPP se traduisent dans les lexèmes construits. Le sens construit des dénominaux se définit directement à partir de celui du $\mathrm{Nc}_{\mathrm{Hum}}$ base. Les déonomastiques s'interprètent en contexte, et renvoient soit directement à un sens dénominatif, soit font intervenir, dans le calcul de leur contenu sémantique, des connaissances, souvent stéréotypiques, liées au référent. La bipartition sémantique des NPP ne relève pas de polysémie mais d'un mécanisme extra-linguistique.

Les distinctions établies entre $\mathrm{Nc}_{\mathrm{Hum}}$ et NPP ne semblent pas s'appliquer à l'ensemble des noms communs et noms propres. Les toponymes par exemple, ont une composante formelle plus proche des noms communs mais le sens des dérivés sur toponyme peut convoquer des informations référentielles (cf. NÉODOMIEN ${ }_{\mathrm{Nc}}$ et BALKANISATION $\mathrm{Nc}_{\mathrm{Nc}}$ ). La classe nominale doit être considérée comme un ensemble très hétérogène de lexèmes disposant de propriétés phonologiques, syntaxiques et sémantiques différentes. Dans notre étude, ces propriétés varient selon la nature du/des référent(s).

\section{Bibliographie}

Allerton, D.J. (1987). The linguistic and sociolinguistic status of proper names. What are they, and who do they belong to? Journal of Pragmatics 11, 1, 61-92.

Anderson, J. M. (2007). The Grammar of Names. New York: Oxford University Press Inc.

Apresjan, J. (1974). Regular Polysemy. Linguistics 142, 5-32.

Baker, M. C. (2003). Lexical categories - Verbs, Nouns, and Adjectives. Cambridge: Cambridge University Press.

Barque, L. (2008). Description et formalisation de la polysémie régulière du français. Thèse de doctorat, Université de Paris 7.

Blevins, J. P. (2006). Word-based morphology. Journal of Linguistics 42, 531-573.

Bonami, O. \& Boyé, G. (2003). Supplétion et classes flexionnelles. Langages 152, 102-126.

Bonami, O., Boyé, G. \& Kerleroux, F. (2009). L'allomorphie radicale et la relation flexion-construction. Aperçus de morphologie. Saint-Denis : Presses Universitaires de Vincennes, 103-126.

Boyé, G. \& Plénat, M. (2014). L'allomorphie radicale dans les lexèmes adjectivaux en français. Le cas des adverbes en -ment. Understanding Allomorphy: Perspectives from OT. London: Equinox Publishing.

Carstairs-McCarthy, A. (2000). Inflection classes. Morphology: A Handbook on Inflection and Word Formation. Berlin: Mouton de Gruyter, 630-637.

Coseriu, E. (1955). El plural en los nombres proprios. Revista Brasileira de filologia 1, 1-15.

Croft, W. (1991). Syntactic categories and grammatical relations: the cognitive organization of information. Chicago, London:University of chicago Press.

Cruse, D. A. (1988). Word meaning and encyclopedic knowledge. Linguistische Arbeiten 210. Tübingen: Niemeyer, 73-84.

Dal, G. \& Namer, F. (2016). À propos des occasionnalismes. SHS Web of Conferences 27.

Flaux, N. \& Van de Velde, D. (2000). Les Noms en français : esquisse de classement. Paris : Ophrys.

Fradin, B. (2003). Nouvelles approches en morphologie. Paris : Presses Universitaires de France.

Gary-Prieur, M.-N. (1971). La notion de connotation(s). Littérature 4, 96-107.

Gary-Prieur, M.-N. (1994). Grammaire du Nom propre. Paris : Presses Universitaires de France.

Gary-Prieur, M.-N. (2016). Le nom propre comme catégorie de la grammaire. Langue française 190, 2, 45-64.

Grevisse, M. \& Goosse, A. (2007). Le Bon Usage. Paris : De Boeck, Duculot.

Hohenhaus, P. (2005). Lexicalization and Institutionalization. Handbook of Word-Formation. Berlin, Dordrecht, Heidelberg, Norwell: Springer, 353-373. 
Hough, C. \& Izdebska D. (2016). Names and Their Environment. Proceedings of the 25th International Congress of Onomastic Sciences, Glasgow, 15-19 August 2014. Glasgow: University of Glasgow. Jespersen, O. (1924). The philosophy of grammar. London: Allen \& Uwin.

Journet-Diallo, O. (2001). Noms d'ancêtres, noms d'amis, noms de dérision. Exemples africains. Spirale 19, 51-60.

Kleiber, G. (1981). Problèmes de référence : descriptions définies et noms propres. Paris : Klincksieck.

Kleiber, G. (1999). Problème de sémantique. La polysémie en question. Villeneuve d'Asq : Presses Universitaires du Septentrion.

Kripke, S. (1980). Naming and Necessity. Oxford UK: Blackwell Publishers.

Langendonck, V. W. (2007). Theory and typology of proper names. Berlin: Mouton de Gruyter.

Lasserre, M. (2016). De l'intrusion d'un lexique allogène. L'exemple des éléments néoclassiques. Thèse de doctorat, Université de Toulouse - Jean Jaurès.

Leroy, S. (2004). Le nom propre en français. Paris : Ophrys.

Leroy, S. (2005). Les dérivés de noms propres dans le TLFi : quelles bases pour quels sens ? Corela [En ligne], HS-1, URL: http://corela.revues.org/1146.

Leroy, S. (2008). Les noms propres et la dérivation suffixale. Neuphilologische Mitteilungen 109, $55-71$.

Lignon, S. \& Namer, F. (à paraître). Catégories d'humains entre dictionnaire et usage : le wagnérien est-il un partisan ou un spécialiste ? Les noms d'humain : théorie, méthodologie et classification. Berlin : De Gruyter.

Lignon, S. \& Roché, M. (2011). Entre histoire et morphophonologie, quelle distribution pour -ien vs -éen ? Des Unités Morphologiques au Lexique. London : Hermès Sciences Publishing, 191-250.

Lignon, S. (2000). La suffixation en -ien-Aspects sémantiques et phonologiques. Thèse de doctorat, Université de Toulouse II - Le Mirail.

Lignon, S. (2002). L'adjectif en -ien comme révélateur de phénomènes de concurrence. BULAG 27, 135- 150.

Lipka, L. (2000). Word-formation and (proper) names: a neglected field. Words: Structure, Meaning, Function, Trends in Linguistics 130, 187-203.

Lyons, J. (1978). Éléments de sémantique. Paris : Larousse Université.

Marin, R. \& Villoing, F. (2012). Lexique 20. Villeneuve d'Asq : Presses Universitaires de Septentrion.

Matthews, P. (1974). Morphology. Cambridge: Cambridge University Press.

Matushansky, O. (2008). On the linguistic complexity of proper names. Linguistics and Philosophy 31, 5, 573-627.

McKinsey, M. (2010). Understanding proper names. Linguistics and Philosophy 33, 4, 325-345.

Mill, J. S. (1843). Système de logique déductive et inductive. Paris: Librairie philosophique de Ladrange.

Mugdan, J. (2015). Units of word-formation. Word formation An International Handbook of the langages of Europe 1. New York, Berlin: Mouton de Gruyter.

Namer, F. (2013). Adjectival bases of French -aliser and -ariser verbs: syncretism or under-specification? In: N. Hathout, F. Montermini \& J. Tcheng (ed.), Selected Papers from the 7th Décembrettes. München: Lincom Europa,185-210.

Noailly, M. (1995). Nom propre et nomination. Paris : Klincksieck.

Plénat, M. \& Roché, M. (2014). Le jeu des contraintes dans la sélection du thème présuffixal. SHS Web of Conferences 8, 1863-1878.

Plénat, M. (2008). Le thème L de l'adjectif et du nom. CMLF 2008.

Plénat, M. (2011). Enquête sur divers effets des contraintes dissimilatives en français. Des Unités Morphologiques au Lexique. London : Hermès Sciences Publishing, 145-190.

Pulgram, E. (1954). Theory of Names. Berkeley, California: American Name Society.

Rauh, G. (2010). Syntactic Categories - Their identification and description in Linguistic Theories. New York : Oxford University Press.

Reboul, A. (1993). Le poids des pères, le choc des fils : prédicats de phase, modificateurs et identification. Cahiers de linguistique française 14, 229-246.

Schnedecker, C. \& Mihatsch, W. (à paraître). Les noms d'humain : théorie, méthodologie et classification. Berlin : De Gruyter.

Schulcket, B. \& Ackermann, T. (2017). The morphosyntax of proper names - An overview. Folia Linguistica 51, 2, 309-339. 
Schweickard, W. (1992). Deonomastik. Ableitungen auf der Basis von Eigennamen im Französischen (unter vergleichenden Berücksichtigung des Italienischen, Rumänischen und Spanischen). Tübingen : Niemeyer.

Strawson, P. F. (1950). On referring. Mind 59, 320-344.

Stump, G. (2005). Word-formation and inflectional morphology. Handbook of Word-Formation. Dordrecht: Springer, 49-72.

Tribout, D., Barque, L., Haas, P. \& Huyghe, R. (2014). De la simplicité en morphologie. SHS Web of Conferences 8.

Vaxelaire, J.-L. (2005). Les noms propres - Une analyse lexicologique et historique. Paris: Honoré Champion.

Villoing, F. (2012). Contraintes de taille dans les mots composés : quand la phonologie entre en concurrence avec les contraintes morphologiques. SHS Web of Conferences 1, 1425-1440.

${ }^{1}$ Par convention, les lexèmes sont notés en petites majuscules et les mots-formes en italique (cf. Matthews, 1974). Nous utilisons NPr, Nc, V, Adj, Adv, pour indiquer les catégories syntaxiques : nom propre, nom commun, verbe, adjectif et adverbe.

${ }^{2}$ Les contextes contenant des dénominaux sont issus du fr WaC et ceux contenant des déonomastiques appartiennent à notre base de données (cf. §3).

${ }^{3}$ La notion de désignateur rigide s'applique difficilement dans les cultures où l'on change de prénom suivant les âges de la vie (cf. Journet-Diallo, $2001: 18$ ).

${ }^{4}$ Cette date est choisie pour qu'il existe des créations lexicales sur les NPP concernés sur la Toile.

${ }^{5}$ L'astérisque symbolise une suite de caractères quelconques.

${ }^{6}$ Data Observer (www.data-observer.com) est une startup spécialisée dans la collecte, le traitement et l'analyse des données issues du Web. Les requêtes ont été effectuées entre janvier 2015 et 2017.

${ }^{7} \mathrm{~S}$ 'il n'y a pas de thème spécifique, le thème par défaut est identique au thème 1.

${ }^{8} \mathrm{Il}$ est sûrement possible d'ajouter un ou plusieurs thèmes à l'espace thématique des anthroponymes tel que nous l'avons défini. On peut, par exemple, penser à la forme /tõtõ/ (surnom de François Mitterrand) utilisée dans le dérivé TONTONISERv.

${ }^{9}$ Le locuteur fait référence aux excuses répétées présentées par Ségolène Royal. En 2008, elle s'excuse plusieurs fois pour des propos de Nicolas Sarkozy. 